\title{
ERRATUM
}

\section{Visualizing nodal heavy fermion superconductivity in $\mathrm{CeColn}_{5}$}

Brian B. Zhou, Shashank Misra, Eduardo H. da Silva Neto, Pegor Aynajian, Ryan E. Baumbach, J. D. Thompson, Eric D. Bauer and Ali Yazdani

Nature Physics 9, 474-479 (2013); published online 14 July 2013; corrected after print 11 September 2013.

In the version of this Letter originally published, the citations of Fig. 1 were incorrect throughout the text. These errors have now been corrected in the HTML and PDF versions of the Letter. 